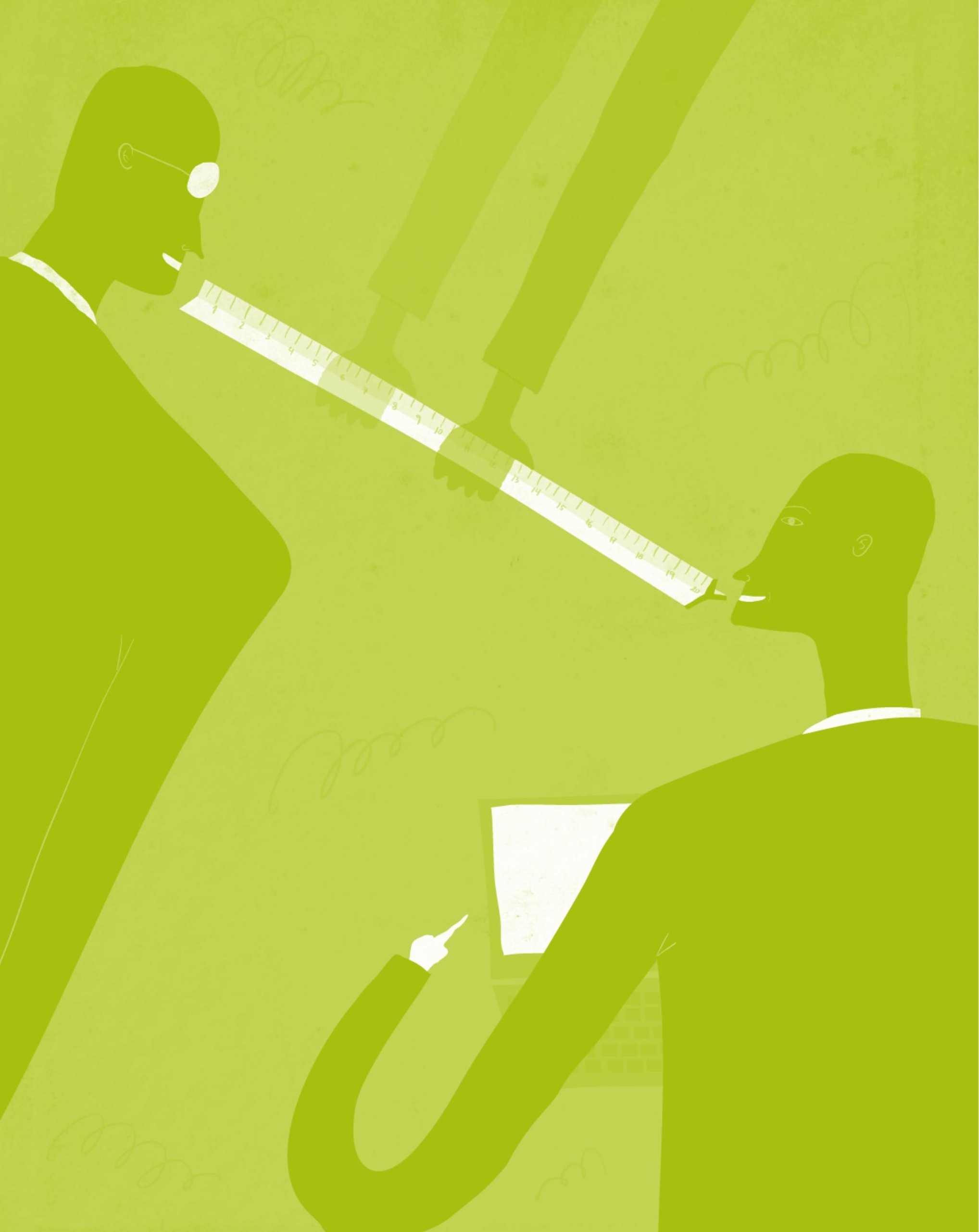




\section{A mensuração do relacionamento organizacional: construção e validação de uma escala oriunda de duas áreas do conhecimento humano}

Gerson José Bonfadini

- Doutor em Comunicação Social pela Pontifícia Universidade Católica do Rio Grande do Sul (PUC-RS)

- Mestre em Desenvolvimento Regional pela Universidade de Santa Cruz do Sul (Unisc)

- Especialista em Marketing pela Universidade Federal do Rio Grande do Sul (UFRGS)

- Graduado em Administração pela UFRGS

- Professor de Marketing da Univates

-bonfa@univates.br

Sandro Luis Kirst

- Doutorando em Comunicação Organizacional pela PUC-RS

- Mestre em Comunicação Organizacional pela PUC-RS

- Especialista em Gestão Universitária pela Univates

- Graduado em Relações Públicas e Administração de Empresas pela UFRGS

- Professor dos cursos de Comunicação na Univates

- slkirst@gmail.com 


\section{Resumo}

O estudo analisa a construção e a validação da escala de relacionamento organizacional, proposta por Bonfadini (2007). A escala resulta da integração de estudos sobre relacionamento organizacional oriundos de duas áreas do conhecimento humano, Relações Públicas e Marketing. A escala integrativa é do tipo multi-itens, formada pelos construtos: comprometimento, confiança, cooperação, poder e satisfação.

PALAVRAS-CHAVE: RELACIONAMENTO ORGANIZACIONAL • ESCALA MULTI-ITENS • RELAÇÕES PÚBLICAS • MARKETING

\section{Abstract}

The study analyzes the construction and validation of the scale of organizational relationship, as proposed by Bonfadini (2007). The scale results from the integration of organizational relationship studies arising from two areas of human knowledge, Public Relations and Marketing. The integration scale is of the multi-item kind, consisting of the following elements: commitment, trust, cooperation, power and satisfaction.

KEYWORDS: ORGANIZATIONAL RELATIONSHIP • MULTI-ITEM SCALE • PUBLIC RELATIONS • MARKETING

\section{Resumen}

El estudio analiza la construcción y la validación de la escala de relación organizacional, propuesta por Bonfadini (2007). Dicha escala resulta de la integración de estudios sobre relaciones organizativas, cuyo origen remite a dos áreas del conocimiento humano: Relaciones Públicas y Marketing. La escala integradora es del tipo multielementos y está compuesta por los constructos: compromiso, confianza, cooperación, poder y satisfacción.

PALABRAS CLAVE: RELACIÓN ORGANIZACIONAL • ESCALA DE MULTI-ARTÍCULO • RELACIONES PÚBLICAS • MARKETING 
| ma organização, conforme Schein (1980, p. 26), “... é composta de duas ou mais pessoas que interagem entre si, por meio de relações recíprocas, para atingir objetivos comuns ${ }^{1 \text { ". }}$. Ao procurar atingir seus objetivos, a organização se depara com os interesses dos diversos públicos que a envolvem. Esses públicos agem no âmbito interno, como funcionários ou gestores, trabalhando e sobrevivendo dos ganhos auferidos; ou no âmbito externo, como, por exemplo, os clientes, que compram os produtos, os fornecedores, que entregam os insumos, ou a comunidade, que vive em sua periferia. Todas essas relações influenciam, direta ou indiretamente, no objetivo organizacional, pois são resultantes do convívio de pessoas e grupos, como observam Mardsen e Townley (2001, p. 37): “A 'organização' é uma abstração dos indivíduos e das relações sociais entre eles que se constitui nessa forma ordenada de vida social".

As relações mediadas pelas organizações crescem, exponencialmente, instigando processos de cooperação ou situações de conflito que influenciam na realização dos objetivos. Atualmente, as organizações, em especial as do tipo empresarial, ultrapassam suas fronteiras de influência, promovendo novas relações com a sociedade (ENRIQUEZ, 2000). Essas relações não se limitam à mera dependência econômica, do tipo trabalho, salário ou benefícios sociais, mas, sim, interferem em aspectos psicossociais como a cultura dos povos, pela disseminação de valores como a competitividade, o consumo e o sucesso.

O relacionamento organizacional, ou seja, o relacionamento que uma organização mantém com os seus diferentes públicos, é objeto de pesquisa deste artigo. O tema relacionamento organizacional é estudado por áreas tão diversas quanto Sociologia, Economia ou Comunicação, que têm gerado diferentes conceitos, definições e tipologias que procuram entender e qualificar o processo de troca que as organizações estabelecem com os seus públicos. Cada área, dentro de seu viés característico de pesquisa, desenvolve saberes tanto técnicos quanto científicos, dando relevância à análise e à avaliação das relações entre as organizações e seus públicos. Esses conhecimentos têm elementos que, ao serem analisados comparativamente, se completam ou se contrapõem. Logo, uma análise comparativa do tema relacionamento organizacional, baseada na integração de elementos teóricos provindos das áreas de Relações Públicas e de Marketing, revela-se importante devido ao ineditismo da abordagem dentro do contexto de estudos interdisciplinares na área de Comunicação Social.

1 Kunsch (2003), na obra Planejamento de relações públicas na comunicação integrada, analisa a importância das organizações na vida das pessoas (sociedade organizacional) e apresenta um estudo detalhado sobre o conceito de organização, bem como do termo "instituição". 
A MENSURAÇÃO DO RELACIONAMENTO ORGANIZACIONAL: CONSTRUÇÃO E VALIDAÇÃO DE UMA ESCALA ORIUNDA DE DUAS ÁREAS DO CONHECIMENTO HUMANO • GERSON JOSÉ BONFADINI • SANDRO LUIS KIRST

\section{0 relacionamento organizacional e a área de Relações Públicas}

As relações estabelecidas por indivíduos de diferentes grupos são o objeto de estudo de diversos autores da área de Relações Públicas. Esse relacionamento entre as diferentes partes só é possível por meio da comunicação, como afirma D’Azevedo (1971). A autora, no início da década de 70 , já destacava a importância da comunicação como um processo de diálogo entre as partes envolvidas, cabendo à área buscar a compreensão mútua entre os envolvidos. Logo, a finalidade da comunicação não é somente transmitir mensagens, mas, sim, estabelecer um caminho de duplo sentido em que emissor e receptor se revezam no processo de comunicação.

Grunig e Hunt (1983) reforçam a idéia de que o relacionamento entre uma organização e seus diferentes públicos se dá por meio da comunicação e o gerenciamento dessa comunicação está a cargo da área de Relações Públicas. Os profissionais da área gerenciam, planejam e executam a comunicação das organizações de forma integral, ou seja, sob uma perspectiva sistêmica. As organizações estabelecem uma comunicação de mão-dupla que possibilita a compreensão mútua entre elas e seus públicos. Com base nessa concepção, os autores afirmam que a atividade de Relações Públicas é um subsistema organizacional que gerencia a comunicação entre a organização e seus públicos, e que deriva do crescente impacto que as organizações e seus públicos causam um ao outro.

As ações desenvolvidas pelas organizações promovem diferentes situações que muitas vezes geram problemas, como poluição, produtos de baixa qualidade ou questões trabalhistas e, como conseqüência desses atos, os públicos podem protestar publicamente, não comprar os produtos e promover greves, gerando problemas para as duas partes. Procurando evitar ou resolver problemas de relacionamento entre as partes, a área de Relações Públicas estabelece um processo de comunicação de mão-dupla, intermediando as relações entre públicos, administração e suas expectativas e objetivos mútuos (GRUNIG e HUNT, 1983).

Simões (1993 e 1995) analisa a relação das organizações com os seus respectivos públicos pelo exercício do poder entre ambos. A construção do referencial teórico do autor está baseada nas relações políticas, no caso, micro políticas, que as organizações estabelecem com os seus públicos. A rede teórica apresenta os conceitos de Relações Públicas dentro de duas abordagens, como ciência e como atividade (SIMÕES, 1995).

Dentro dessa ótica, o autor afirma que o conflito é iminente nessa relação. Logo, a relação no sistema organização-públicos estabelece diferentes níveis: interesses satisfeitos (organização e públicos se relacionam bem), passando pela insatisfação, boatos, conflito, crise, podendo chegar até à convulsão social (uso da violência decorrente do antagonismo extremo entre as partes). Assim, administrar a função política de uma organização é uma atividade estratégica, pois a organização, por meio de suas políti- 
cas e normas, procura alcançar benefícios comuns no sistema organização-públicos, legitimando a relação e solucionando os conflitos.

A atuação estratégica da área de Relações Públicas no âmbito organizacional é um dos elementos centrais da abordagem teórica de Kunsch (1997 e 2003). A autora, na obra Relações públicas e modernidade, de 1997, destaca que a comunicação de uma organização deve ser integrada, ou seja, deve reunir diferentes compostos que formam a comunicação nas organizações (institucional, mercadológica, interna e administrativa) para atuarem de forma sinérgica e dentro de uma política desenvolvida para toda a organização. Essa integração da comunicação permite a conformação da identidade corporativa perante os diferentes públicos, a administração equilibrada da comunicação entre a organização e seus públicos e a atuação estratégica da área de Relações Públicas no contexto organizacional. Kunsch (2003) desdobra o conceito de comunicação integrada dentro do contexto do planejamento estratégico. Apresenta as diferentes etapas da construção do planejamento estratégico, integrando a comunicação nas organizações por meio da atribuição de responsabilidade à área de Relações Públicas no foco de planejar, administrar e pensar estrategicamente a sua comunicação.

Andrade (2001), ao relacionar as diferentes definições sobre a área de Relações Públicas, destaca que não há consenso sobre tal. Com base nessa constatação, o autor desenvolve um processo que busca alcançar o objetivo de Relações Públicas, que é: “... transformar os diversos espectadores, clientes e funcionários da organização em autênticos públicos, por meio de ampla liberdade de informação e discussão” (p. 89). O processo proposto por Andrade reside em desenvolver seis fases distintas, a saber:

- $1^{\text {a }}$ fase - determinação do grupo e sua identificação como público: consiste em conhecer os grupos que direta ou indiretamente se relacionam com a organização;

- $2^{\mathrm{a}}$ fase - apreciação do comportamento do público: com pesquisas e análises sistemáticas, conhecer as opiniões, comportamentos e posições que os diferentes públicos têm em relação à organização;

- $3^{a}$ fase - levantamento das condições internas: é a análise crítica de normas e métodos de trabalho utilizados na organização, relativos à estrutura, às relações de trabalho, às instalações, entre outros;

- $4^{\mathrm{a}}$ fase - revisão e ajustamento da política administrativa: é a revisão e a melhoria de normas e processos para eliminar os problemas e ajustá-los às demandas dos grupos;

- $5^{a}$ fase - programa de informações: é a preparação e a veiculação de material informativo que estimule o interesse dos públicos em relação à organização;

- $6^{a}$ fase - controle e avaliação dos resultados: é o feedback do processo, ou seja, é a retroalimentação das impressões que os públicos tiveram após todo o trabalho desenvolvido, servindo de base para ações corretivas. 
A MENSURAÇ̃̃O DO RELACIONAMENTO ORGANIZACIONAL: CONSTRUÇÃO E VALIDAÇÃO DE UMA ESCALA ORIUNDA DE DUAS ÁREAS DO CONHECIMENTO HUMANO • GERSON JOSÉ BONFADINI • SANDRO LUIS KIRST

A abordagem sistêmica também é utilizada por Fortes (2003). Ele afirma que a sociedade atual se caracteriza por um ambiente que privilegia o conhecimento e a informação, devido às mudanças e às evoluções tecnológicas. O autor reforça que as organizações que procuram obter êxito em seus empreendimentos devem consolidar os sistemas de relacionamentos com públicos. Para Fortes, o sistema é gerenciado pela área de Relações Públicas, que tem conhecimento necessário para desenvolver e implementar políticas de comunicação entre as organizações e seus diversos públicos.

O autor observa que, para implantar um programa de Relações Públicas numa organização, é necessário estabelecer um processo adequado que promova uma ação planejada, fundamentada em pesquisa, na comunicação estratégica e na participação programada. Isso possibilita à área consolidar-se politicamente dentro da organização, sendo vista como o setor capacitado e especializado em estabelecer relacionamentos positivos com os diferentes públicos organizacionais.

Ao identificar a existência de vários públicos que se relacionam com as organizações, Fortes destaca que estes podem, por sua opinião ou comportamento, influenciar no bom desempenho das organizações. Logo, a área de Relações Públicas tem importante papel no relacionamento organizacional. A definição dos públicos de interesse para uma organização deve levar em consideração a interdependência entre as partes, derivada de critérios lógicos de relacionamento, como nos demonstra França (2004). $\mathrm{O}$ autor destaca que o principal objetivo do relacionamento organizacional é o de sustentar "[...] interesses institucionais, promocionais ou de desenvolvimento de negócios como sucede com os colaboradores, clientes, fornecedores, revendedores e demais públicos ligados às operações produtivas e comerciais da organização". (FRANÇA, 2004, p. 100)

Os estudos de Grunig e Hon (1999) estabelecem os fundamentos para a mensuração do relacionamento entre uma organização e os diferentes públicos de interesse, dentro da área de Relações Públicas. Os pesquisadores destacam que diferentes técnicas e ferramentas já foram elaboradas para avaliar as ações de Relações Públicas, mas essas se concentram em horizontes de tempo de curto prazo. Por exemplo, técnicas que avaliam a boa imagem da organização, a exposição da organização na mídia, a recepção e retenção da mensagem pelo público, entre outros, possibilitam avaliar de forma pontual, mas não mensuram o relacionamento da organização com seus diferentes públicos de forma global:

"Relações Públicas tornam uma organização mais efetiva quando identificam os principais públicos estratégicos dentro de um processo de gestão estratégica e conduzem os programas de comunicação a desenvolver e manter, de forma efetiva, relacionamentos de longo prazo entre a administração e seus públicos". (GRUNIG e HON, 1999, p. 12)

Logo, os autores sugerem o desenvolvimento de um instrumento que avalie o relacionamento organizacional de longo prazo. 
Os pesquisadores desenvolveram uma escala que mensura o relacionamento organizacional, no longo prazo, denominada PR Relationship Measurement Scale. O estudo selecionou cinco atributos dessa escala: controle recíproco, confiança, satisfação, comprometimento e relacionamento comunal.

O controle recíproco é quando as partes envolvidas concordam que cada uma tem o legítimo poder de influenciar à outra (GRUNIG e HON, 1999 e YANG e GRUNIG, 2005). Mesmo que haja um desequilíbrio natural na relação entre a organização e seus públicos (assimetria de poder), urge a necessidade de que cada uma das partes tenha algum controle sobre a outra, para que ocorra uma relação estável (HON e BRUNNER, 2002).

A confiança, conforme Grunig e Hon (1999), está relacionada à confiabilidade que as partes têm de revelarem-se uma à outra. Há três dimensões na confiança: integridade ou crença de que a organização é justa; coerência ou crença de que a organização fará aquilo que ela diz que fará; e competência ou crença de que a organização tem a habilidade para fazer aquilo que diz que fará. Confiança e comprometimento destacam-se nos estudos de Relações Públicas, pois são consideradas dimensões ${ }^{2}$ do relacionamento da organização e seus públicos (LEDINGHAM e BRUNING, 1998 e HUNG, 2005).

O atributo satisfação avalia o quanto cada parte se sente favorecida pela outra, pois as expectativas positivas reforçam o relacionamento, sendo que um relacionamento satisfatório, da organização com cada um de seus públicos, é um benefício que excede em valor os custos (GRUNIG e HON, 1999). Satisfação pode ser entendida como a medida da extensão na qual os benefícios do relacionamento excedem as expectativas de ambas as partes (JO, HON e BRUNNER, 2004).

O comprometimento é a medida na qual as partes sentem e acreditam que vale a pena gastar energia para manter e expandir o relacionamento. Tem duas dimensões: comprometimento contínuo, relativo a uma determinada linha de conduta, e comprometimento afetivo, derivado de uma orientação emocional (GRUNIG e HON, 1999). Tanto a organização quanto os públicos procuram direcionar as emoções e o comportamento para assegurar que o relacionamento perdure (HUNG, 2005).

Relacionamento comunal se caracteriza pela ação que cada uma das partes faz ao ajudar a outra, pois na relação organização-público, o bem-estar da outra parte deve ser alcançado, até mesmo quando não se obtêm retorno algum da outra parte (GRUNIG e HON, 1999). "Isto implica, até certo ponto, quanto uma organização está realmente preocupada com o bem-estar dos públicos (e vice-versa). [...] Ou, em outras palavras, se as partes envolvidas têm atitudes altruistas." (JO, HON e BRUNNER, 2004, p. 17)

2 As cinco dimensões são: confiança, abertura, participação, investimento e comprometimento. 
A MENSURAÇÃO DO RELACIONAMENTO ORGANIZACIONAL: CONSTRUÇÃO E VALIDAÇÃO DE UMA ESCALA ORIUNDA DE DUAS ÁREAS DO CONHECIMENTO HUMANO • GERSON JOSÉ BONFADINI • SANDRO LUIS KIRST

\section{0 relacionamento organizacional e a área de Marketing}

A teoria de Marketing que sedimenta o relacionamento organizacional é a de Marketing de Relacionamento, a qual apresenta uma ampla e profunda gama de pesquisas, tendo um variado número de construtos que dão a dimensão da complexidade e amplitude do tema. Durante as últimas décadas, diversos autores, como Morgan e Hunt (1994), Ganesan (1994), Grönross (1994), Wilson e Vlosky (1997) e Fontenot e Wilson (1997), identificaram e analisaram diversas tipologias que qualificaram a pesquisa acadêmica pela correlação entre os construtos e da elaboração de modelos preditivos para a área.

A Commitment-Trust Theory, desenvolvida por Morgan e Hunt (1994), apresenta a Key Mediating Variable (KMV) como modelo que possibilita avaliar o marketing de relacionamento de uma organização com os seus diferentes parceiros. Como argumentam os autores: "[...] compromisso e confiança resultam, diretamente, em atitudes cooperativas que são propícias para o sucesso do marketing de relacionamento" (MORGAN e HUNT, 1994, p. 03), construindo um relacionamento de longo prazo, evitando o comportamento oportunista. Os três primeiros construtos analisados no estudo são: o comprometimento, a confiança e a cooperação.

O comprometimento é elemento central no processo de compra dos consumidores (GARBARINO e JOHNSON, 1999), pois estimula o desejo de um relacionamento duradouro (MOORMAM, ZALTMAN e DESHPANDE, 1992) e do cumprimento das promessas estabelecidas nas trocas mútuas entre a empresa e seus diferentes parceiros (GRÖNROOS, 1994).

A confiança está centrada na credibilidade que cada uma das partes tem perante a outra (SIRDESHMUKH, SINGH e SABOL, 2002), promovendo a lealdade nas trocas relacionais (NOOTEBOOM, BERGER e NOODERHAVEN, 1997) e, dentro da prestação de serviços, se constitui em um poderoso instrumento de marketing de relacionamento empresarial (BERRY, 1995).

Já a cooperação é o paradigma que deriva diretamente dos construtos centrais de comprometimento e confiança (MORGAN e HUNT, 1994), caracterizando-se por ações conjuntas e coordenadas entre os parceiros de troca que procuram atingir um objetivo em comum (ANDERSON e NARUS, 1990). Como os consumidores são a parte mais fraca nas relações estabelecidas com as empresas, procuram formar relações mais duradouras, eliminando a assimetria de poder no processo de troca (GANESAN, 1994).

Fontenot e Wilson (1997) relacionaram uma extensa lista de construtos criados e desenvolvidos por diversos autores. Dentre esses, o estudo destaca os paradigmas poder e satisfação. 
Poder é a capacidade que um dos parceiros de troca tem de influenciar na decisão do outro (FONTENOT e WILSON, 1997). É elemento central dentro das dimensões que envolvem controle ou dominância de pessoas e recursos (SCHWARTZ, 1994), influenciando no nível de cooperação entre as partes na troca relacional (JOSHI E STUMP, 1999).

A satisfação é o paradigma que avalia o sentimento resultante da comparação entre o que se obteve e o que era esperado (FONTENOT e WILSON, 1997). Dentro do processo de consumo de um produto ou serviço, está atrelada à avaliação de eventos, como tempo de espera, concessões e conforto, e aos resultados do consumo, como eficiência, eficácia, riscos menores, sucesso, entre outros (OLIVER, 1997), levando o usuário a considerar e valorizar não somente o produto ou serviço em si, mas a sua interação e experiência com o consumo (HOLBROOK, 1994).

\section{Procedimentos metodológicos}

Os procedimentos metodológicos que orientaram o estudo na construção, avaliação e validação da escala derivam da tese de doutorado desenvolvida por Bonfadini (2007). $\mathrm{O}$ artigo avança na proposta da tese por meio da aplicação do framework criado por Churchill (1979). Esse instrumento metodológico revelou-se apropriado, pois possibilitou a validação de paradigmas e medição da escala multi-itens (MALHOTRA, 1996).

A primeira etapa, conforme Churchill (1979), elaborar a teoria ${ }^{3}$, estabelece a teoria subjacente do constructo avaliado que possibilita elaborar a escala e interpretar os escores resultantes (MALHOTRA, 1996). A base teórica, revista e ampliada, está descrita nos itens anteriores do artigo.

A segunda etapa, gerar conjunto inicial de itens, consistiu na geração do conjunto inicial de itens da escala com base na análise da teoria, de dados secundários e da pesquisa qualitativa. Essa análise está detalhada na tese de Bonfadini (2007) e utilizou a técnica de análise de conteúdo (BARDIN, 1988 e MALHOTRA, 1996). O estudo do corpus teórico possibilitou a identificação das categorias (atributos e construtos) em comum nas pesquisas sobre relacionamento organizacional nas áreas de Relações Públicas e de Marketing. As categorias que geraram o conjunto inicial de itens analisados no estudo são: comprometimento, confiança, cooperação, poder e satisfação.

A terceira etapa, escolher um conjunto reduzido de itens, definiu as variáveis derivadas das categorias relacionadas na etapa anterior. A definição, também, está detalhada em Bonfadini (2007), e derivou da aplicação da técnica de grupo focal, com base na teo-

3 A denominação das etapas utiliza a tradução livre adotada na obra de Malhotra (2001). 
A MENSURAÇÃO DO RELACIONAMENTO ORGANIZACIONAL: CONSTRUÇÃO E VALIDAÇÃO DE UMA ESCALA ORIUNDA DE DUAS ÁREAS DO CONHECIMENTO HUMANO • GERSON JOSÉ BONFADINI • SANDRO LUIS KIRST

ria proposta por Rea e Parker (2002) e Costa (2005), gerando as variáveis (itens) provindas das contribuições dadas por estudantes e professores das áreas de Relações Públicas e de Marketing de uma universidade no Sul do Brasil. Foram elaboradas três variáveis representativas de cada uma das categorias escolhidas ${ }^{4}$. Logo, foram criadas quinze variáveis para cada uma das áreas estudadas, totalizando uma escala multi-itens de trinta variáveis. As variáveis foram construídas com base na análise de conteúdo das sugestões provindas dos grupos focais em consonância com os elementos teóricos propostos nas áreas de Relações Públicas e de Marketing (BONFADINI, 2007).

As demais etapas de avaliação e validação da escala de relacionamento organizacional proposta pelo estudo estão desenvolvidas abaixo.

\section{Etapa 4: coletar dados de uma amostra pré-teste}

A quarta etapa, proposta por Churchill (1979), testou, de forma qualitativa, a validade das variáveis elaboradas para a escala de relacionamento organizacional construída por Bonfadini (2007) com base na validade nominal do conteúdo das variáveis.

A avaliação subjetiva das variáveis foi feita dentro dos seguintes parâmetros:

A primeira avaliação foi a de método de juizes, acadêmicos e práticos (MALHOTRA, 2001). Realizada por seis especialistas, dois acadêmicos e um profissional de cada uma das áreas envolvidas no estudo, ou seja, três especialistas de Relações Públicas e três especialistas de Marketing criticaram e apresentaram sugestões em relação à validade técnica do questionário.

A segunda avaliação foi baseada em um pré-teste, ou seja, na aplicação a campo da escala, usando-se um esboço do instrumento de pesquisa, do tipo questionário, num grupo, escolhido por conveniência, de quarenta pessoas. Esse grupo se caracterizou por ser composto de quinze alunos e cinco professores dos cursos de Administração e quinze alunos e cinco professores da área de Comunicação Social, revelando consonância à proposta de Malhotra (2001, p. 291): "Em geral, o tamanho da amostra do pré-teste é pequeno, variando de 15 a 30 entrevistados para o teste inicial, dependendo da heterogeneidade da população visada”. As contribuições, críticas e sugestões do grupo possibilitaram validar o conteúdo das variáveis, finalizando a análise qualitativa da escala.

\section{Etapa 5: fazer análise estatística}

A quinta etapa testou, de forma quantitativa, a validade de construto das variáveis elaboradas para a escala de relacionamento organizacional construída por Bonfadini (2007).

4 A saber, comprometimento, confiança, cooperação, poder e satisfação. 
A avaliação conclusiva das variáveis foi feita dentro dos seguintes parâmetros:

A escala foi operacionalizada graças à elaboração de um instrumento de pesquisa, do tipo questionário, que foi construído com as seguintes características: utilizou-se uma escala não-comparativa, do tipo Likert, com perguntas fechadas e pontuação de 1 a 5 , para avaliar o grau de concordância da amostra em relação a cada variável da escala multi-itens; a ordem das variáveis no questionário foi determinada de forma aleatória, procurando diminuir a tendência de viés das respostas em relação a cada categoria analisada; e, os formulários foram devidamente codificados para a adequada tabulação e depuração dos dados.

A determinação da amostra na qual a escala foi avaliada ocorreu dentro dos seguintes critérios: o público escolhido foi o corpo discente da instituição de ensino superior avaliada na tese de Bonfadini (2007); a população de alunos de Comunicação Social e de Administração totalizou 1.783 pessoas, gerando uma amostra confiável ${ }^{5}$ de 477 alunos (MATTAR, 1996); o método de amostragem utilizado foi o probabilístico do tipo aleatório simples, pela escolha dos respondentes com base em tabela de números aleatórios perante uma listagem, em ordem alfabética, de toda a população de alunos.

Após a devida coleta, tabulação e depuração dos dados, a escala foi analisada quanto à confiabilidade e à validade do instrumento, compostas pelas variáveis aplicadas pelo instrumento de pesquisa.

\section{Análise da confiabilidade de consistência interna}

A confiabilidade da escala de relacionamento organizacional foi medida com base na abordagem de consistência interna. Avaliou-se a escala multidimensional, representada por cada uma das categorias, pela soma dos itens que formam o escore geral ${ }^{6}$, medindo os escores meio a meio. Esses escores foram mensurados pelo Coeficiente Alfa (a) para cada uma das categorias que estruturam o instrumento de pesquisa, calculando a confiabilidade de consistência interna para cada categoria, como sugere Malhotra (2001).

A Tabela 1 apresenta os resultados obtidos no cálculo da confiabilidade interna do instrumento. Cabe lembrar que, para ser considerado satisfatório, o coeficiente alfa deve apresentar resultado maior ou igual a 0,6, numa escala de zero a um. Em negrito, são apresentados os resultados do Alfa de Cronbach de cada uma das cinco categorias avaliadas no estudo. Todos os coeficientes são estatisticamente significativos, em nível inferior a 0,001 .

$595 \%$ de nível de confiança e um erro amostral máximo de 5\%.

6 É a média das trinta variáveis construídas para o instrumento de pesquisa. 
A MENSURAÇÃO DO RELACIONAMENTO ORGANIZACIONAL: CONSTRUÇÃO E VALIDAÇÃO DE UMA ESCALA ORIUNDA DE DUAS ÁREAS DO CONHECIMENTO HUMANO • GERSON JOSÉ BONFADINI • SANDRO LUIS KIRST

\section{Tabela 1}

\section{Coeficiente Alfa de Cronbach de cada categoria}

\begin{tabular}{|l|c|c|c|c|c|}
\hline \multicolumn{1}{|c|}{ Categorias } & Comprometimento & Confiança & Cooperação & Poder & Satisfação \\
\hline Comprometimento & $\mathbf{0 , 7 9 1}$ & & & & \\
\hline Confiança & & $\mathbf{0 , 8 2 3}$ & & & \\
\hline Cooperação & & & $\mathbf{0 , 7 9 4}$ & & \\
\hline Poder & & & $\mathbf{0 , 7 4 6}$ & \\
\hline Satisfação & & & & $\mathbf{0 , 7 8 5}$ \\
\hline
\end{tabular}

Fonte: elaborada com base na análise estatística sobre a base de dados da pesquisa.

Os dados apresentados acima demonstram que a confiabilidade de consistência interna do instrumento é satisfatória, ou seja, apresenta Alfa de Cronbach (a) maior ou igual ( $>$ ou =) a 0,6, como indicam Malhotra (2001) e McDaniel e Gates (2003). Por ser um construto, a priori, multidimensional, mediu-se o Coeficiente Alfa para cada uma das categorias (dimensões) escolhidas para a construção do instrumento de pesquisa. Os valores apresentados, mínimo de 0,746 para a categoria poder e máximo de 0,823 para a categoria confiança, demonstram o grau de confiabilidade do instrumento.

\section{Validade de construto da escala de relacionamento organizacional}

A validade de construto da escala proposta foi obtida por meio da validade convergente existente entre as categorias analisadas, do uso da correção para atenuação e da aplicação de análise fatorial.

\section{a) Validade convergente}

A validade convergente do construto estudado deriva da análise estatística obtida pela técnica de Correlação de Pearson ${ }^{7}$, que indicou correlação positiva ${ }^{8}$ e significativa ${ }^{9}$ entre os itens do estudo. Ela indica que existe interdependência entre as variáveis relacionadas às suas respectivas categorias, ou seja, elas sofrem influência de forma direta e progressiva entre si, permitindo deduzir que o construto é válido perante o contexto pesquisado.

7 Criada por Karl Pearson, é conhecida também como Correlação Momento-Produto, Correlação Simples, Correlação Bivariada ou Coeficiente de Correlação (MALHOTRA, 2001 e MCDANIEL e GATES, 2003).

8 Conforme McDaniel e Gates (2003, p. 490): " 0 valor de $R$ (coeficiente de correlação) pode ir de -1 (correlação negativa perfeita) a +1 (correlação positiva perfeita). Quanto mais perto $R$ estiver de + ou -1 , mais forte será o grau de associação entre $X$ e $Y$. Se $R$ for igual a zero, então não haverá associação entre X e Y."

9 Todos os coeficientes são estatisticamente significativos em nível inferior a 0,01. 
Na Tabela 2 são apresentados, na diagonal e em negrito, os dados do Coeficiente Alfa de cada categoria e, fora da diagonal, os coeficientes de correlação entre as categorias.

\section{Tabela 2}

\section{Coeficientes de correlação de Pearson entre as categorias \\ e Coeficientes Alfa de cada categoria}

\begin{tabular}{|l|c|c|c|c|c|}
\hline \multicolumn{1}{|c|}{ Categorias } & Comprometimento & Confiança & Cooperação & Poder & Satisfação \\
\hline Comprometimento & $\mathbf{0 , 7 9 1}$ & $0,778^{* *}$ & $0,801^{* *}$ & $0,737^{* *}$ & $0,781^{* *}$ \\
\hline Confiança & & $\mathbf{0 , 8 2 3}$ & $0,763^{* *}$ & $0,796^{* *}$ & $0,791^{* *}$ \\
\hline Cooperação & & & $\mathbf{0 , 7 9 4}$ & $0,779^{* *}$ & $0,782^{* *}$ \\
\hline Poder & & & & $\mathbf{0 , 7 4 6}$ & $0,719^{* *}$ \\
\hline Satisfação & & & & $\mathbf{0 , 7 8 5}$ \\
\hline
\end{tabular}

Fonte: elaborada com base na análise estatística sobre a base de dados da pesquisa.

** Correlação é significativa ao nível de 0,01 (bi-caudal).

A análise dos dados obtidos aponta que a correlação entre as variáveis das categorias que compõem a escala desenvolvida é positiva entre as categorias analisadas, apresentando resultados entre 0,719 (Satisfação e Poder) até 0,801 (Comprometimento e Cooperação), sendo que a correlação entre as categorias é significativa ao nível de 0,01 .

\section{b) Correção para atenuação}

A correção para atenuação ${ }^{10}$ permite analisar o grau de correlação e interdependência entre as categorias analisadas no estudo, podendo ser utilizada na determinação da validade do construto do instrumento. Logo, a correção para atenuação estima o grau de correlação existente entre os coeficientes corrigidos (desatenuados) de cada categoria, isto é, identifica se o coeficiente de correlação entre duas categorias (coeficiente de fidedignidade ou Coeficiente Alfa) é idêntico à unidade. Isso permite apontar o quanto as variáveis das categorias são correlacionadas "verdadeiramente" entre si, pois, quanto mais próximo da unidade, ou seja, apresentar resultado igual a "1", maior será a sua "verdadeira" interdependência (WHERRY, 1984), validando o construto proposto. Os resultados dos coeficientes de correlação entre as categorias, corrigidos para atenuação, estão descritos na tabela a seguir.

Como todos os coeficientes de correlação corrigidos são muito próximos da unidade, isso significa que todas as categorias tendem a ser uma medida do mesmo construto e que, portanto, para fins de análise, pode-se trabalhar com um único escore para cada respondente: o escore médio, ou escore geral, nos 30 itens que constituem a escala de relacionamento organizacional, validando o construto.

10 Conforme Wherry (1984), é uma técnica estatística que permite estimar qual seria o coeficiente de correlação entre duas variáveis, caso as medidas dessas variáveis não tivessem erro; dessa forma, a correção para atenuação permite estimar o "verdadeiro coeficiente de correlação". 
A MENSURAÇÃO DO RELACIONAMENTO ORGANIZACIONAL: CONSTRUÇÃO E VALIDAÇÃO DE UMA ESCALA ORIUNDA DE DUAS ÁREAS DO CONHECIMENTO HUMANO • GERSON JOSÉ BONFADINI • SANDRO LUIS KIRST

Tabela 3

Coeficientes de correlação entre as categorias, corrigidos para atenuação

\begin{tabular}{|l|c|c|c|c|c|}
\hline \multicolumn{1}{|c|}{ Categorias } & Comprometimento & Confiança & Cooperação & Poder & Satisfação \\
\hline Comprometimento & $\mathbf{1}$ & 0,964 & 1 & 0,959 & 0,991 \\
\hline Confiança & & $\mathbf{1}$ & 0,944 & 1 & 0,984 \\
\hline Cooperação & & & $\mathbf{1}$ & 1 & 0,991 \\
\hline Poder & & & & $\mathbf{1}$ & 0,940 \\
\hline Satisfação & & & & 1 \\
\hline
\end{tabular}

Fonte: elaborada com base na análise estatística sobre a base de dados da pesquisa.

\section{c) Análise fatorial}

A análise fatorial é um processo estatístico que procura reduzir e sumarizar dados, identificando variáveis inter-relacionadas dentro de parâmetros gerenciáveis. Malhotra (2001, p. 504) afirma que a análise fatorial é uma técnica de interdependência, ou seja, "[...] uma técnica estatística multivariada em que se examina todo o conjunto de relações interdependentes”. A análise fatorial das variáveis do instrumento apresentam apenas as cargas fatoriais superiores a 0,30 , ou seja, o indicador aponta as correlações entre as variáveis estudadas em relação ao construto proposto. O método para gerar os fatores iniciais foi o de componentes principais, retendo fatores com autovalor (eigenvalue) superior a 1. Foram retidos cinco fatores e rotados pelo método Quartimax. Esse método privilegia uma estrutura fatorial com um fator em que a maioria das variáveis aparece e procura gerar os fatores secundários como fatores de grupo de variáveis. Os dados relativos à análise fatorial de cada uma das variáveis (itens) da escala de relacionamento organizacional criada por Bonfadini (2007) estão na Tabela 4.

Conforme se observa na tabela, todos os itens têm carga fatorial importante no primeiro fator. Esse resultado é consistente para a análise desenvolvida na tese com base na consistência interna de cada grupo de itens e, posteriormente, pelas correlações entre os diversos agrupamentos de itens. Dessa forma, a análise fatorial corrobora os resultados obtidos nas análises anteriormente efetuadas.

Portanto, pelas análises estatísticas desenvolvidas no estudo, pode-se afirmar que a escala de relacionamento organizacional apresenta confiabilidade e validade, tanto de conteúdo quanto de construto, sendo representativa do processo de integração de ações oriundas das áreas de Relações Públicas e de Marketing. E como essa escala é composta de categorias e variáveis que representam as ações integradas das áreas, tanto as categorias quanto as variáveis são confiáveis, válidas e representam medida de mesmo construto, podendo se afirmar que representam as ações de relacionamento organizacional que uma organização estabelece com os seus diferentes públicos. 
Tabela 4

Análise fatorial relativa à matriz rotada do fator

\begin{tabular}{|c|c|c|c|c|c|}
\hline Variável & Fator 1 & Fator 2 & Fator 3 & Fator 4 & Fator 5 \\
\hline 01 - Valoriza &, 551 & ,308 & & & \\
\hline 02 - Atitudes & ,537 & ,382 & & & \\
\hline 03 - Espaço & ,472 &, 570 & & & \\
\hline 04 - Competência &, 553 & &, 539 & & \\
\hline 05 - Opinião &, 521 &, 532 & & & \\
\hline 06 - Confiáveis & ,663 & & & & \\
\hline 07 - Profissional &, 572 & & &,- 375 &, 337 \\
\hline 08 - Agradável &, 517 & ,362 & & & \\
\hline 09 - Satisfatório & ,633 & & & & \\
\hline 10 - Respeita & ,515 & &,- 566 & & \\
\hline 11 - Bem-Estar & ,645 & & & & \\
\hline 12 - Esforços & ,628 & & & & ,532 \\
\hline 13 - Perdure & ,676 & & & & \\
\hline 14 - Longo Prazo & ,711 & & & & \\
\hline 15 - Coerente & ,686 & & & & \\
\hline 16 - Benefícios &, 515 & & &, 513 & \\
\hline 17 - Orgulho & 650 & & 337 & ,358 & \\
\hline 18 - Possível & ,685 & & & & \\
\hline 19 - Falam Bem & ,616 & & ,402 & & \\
\hline 20 - Pressão & ,589 & & &,- 312 & \\
\hline 21 - Cooperação & ,716 & & & & \\
\hline 22 - Capacidade & ,703 & & & & \\
\hline 23 - Comprometida & ,643 & & & & \\
\hline 24 - Credencia & ,737 & & & & \\
\hline 25 - Estimula & ,736 & & & & \\
\hline 26 - Valorizo & ,624 & & & & \\
\hline 27 - Decisões & ,663 & & & &,- 309 \\
\hline 28 - Integro & ,692 & & & & \\
\hline 29 - Promete & ,697 & & & & \\
\hline 30 - Parceria & 687 & & & & \\
\hline
\end{tabular}

Fonte: elaborada com base na análise estatística sobre a base de dados da pesquisa.

\section{Etapa 6: elaborar a escala purificada}

A sexta etapa é derivada da validação estatística ocorrida na etapa 5, ou seja, após a análise da escala multi-itens proposta no estudo, as variáveis (itens) são depuradas e eliminadas as categorias e/ou variáveis que não apresentam confiabilidade e validade em relação ao construto analisado. As categorias e variáveis que compõem a escala analisada, validada e purificada neste estudo estão descritas a seguir, na ordem apresentada no questionário aplicado: 
A MENSURAÇÃO DO RELACIONAMENTO ORGANIZACIONAL: CONSTRUÇÃO E VALIDAÇÃO DE UMA ESCALA ORIUNDA DE DUAS ÁREAS DO CONHECIMENTO HUMANO • GERSON JOSÉ BONFADINI • SANDRO LUIS KIRST

1. Tanto a organização " $\mathrm{X}$ " me valoriza como pessoa quanto eu a valorizo como organização.

2. A organização "X" estimula as atitudes de cooperação entre as pessoas.

3. A organização " $X$ " possibilita que pessoas como eu tenham espaço para dar sua opinião.

4. A comunidade em geral tem confiança na competência da organização "X".

5. Na organização " $X$ ", as pessoas podem expressar sua opinião livremente.

6. As ações realizadas tanto por mim quanto pela organização " $X$ ” são confiáveis, pois se caracterizam pela coerência.

7. No relacionamento que nós mantemos, tanto eu quanto a organização "X" temos atitudes profissionais.

8. A organização " $X$ " proporciona um ambiente agradável para estar.

9. O relacionamento estabelecido entre mim e a organização " $X$ " é satisfatório para ambos.

10. A organização " $X$ " respeita as pessoas que têm dificuldades, sejam econômicas ou sociais.

11. As decisões tomadas, tanto por mim quanto pela organização " $X$ ", levam em consideração o bem-estar de ambos.

12. Noto que os esforços feitos, tanto pela organização " $X$ " quanto por mim, buscam o desenvolvimento tanto pessoal quanto profissional.

13. Tanto eu quanto a organização " $X$ " nos esforçamos para que o nosso relacionamento perdure.

14. Tanto eu quanto a organização " $X$ ” consideramos muito importante manter o relacionamento no longo prazo.

15. A organização " $X$ " trata as pessoas de forma coerente e justa.

16. Os benefícios alcançados hoje, tanto por mim quanto pela organização " $X$ ", são maiores que os esperados no início do nosso relacionamento.

17. Os clientes têm orgulho de comprar na organização " $X$ ".

18. A organização " $X$ " se esforça para garantir que o relacionamento com os clientes seja o melhor possível.

19. As pessoas da comunidade que conhecem a organização " $X$ " geralmente falam bem dela.

20. Nunca ocorreram atitudes de pressão ou de indução, tanto minhas quanto da organização " $X$ ", em nosso relacionamento.

21. A cooperação, tanto minha quanto da organização " $X$ ", é uma característica do nosso relacionamento.

22. As ações realizadas, tanto por mim quanto pela organização " $X$ ”, estão baseadas na capacidade e na competência de cada uma das partes.

23. A organização " $X$ " está comprometida em manter um relacionamento de longo prazo comigo.

24. O retorno que eu e a organização " $X$ " temos com o nosso relacionamento credencia-nos a continuá-lo por muitos anos.

25. A organização " $X$ " estimula a cooperação entre ela e os clientes. 
26. Comparando com outras organizações do setor, eu valorizo mais o relacionamento com a organização " $X$ ".

27. A organização " $X$ " toma decisões levando em consideração a opinião dos clientes.

28. O relacionamento entre mim e a organização " $X$ " é integro e honesto, pois cumprimos o que está acordado.

29. A organização " $X$ " tem competência suficiente para realizar o que promete.

30. O ambiente que se encontra nas dependências da organização " $X$ " estimula a parceria e a solidariedade.

A validação da escala em uma primeira rodada de análise estatística perante um determinado público credencia a escala multi-itens apresentada por Bonfadini (2007) a identificar sua confiabilidade e validade perante outros públicos, buscando a generalização da escala em outros estudos.

\section{As demais etapas da elaboração de uma escala multi-itens}

Uma escala multi-itens deve ser validada não somente por uma amostra perante um determinado público: deve ser testada estatisticamente perante outras amostras visando a sua purificação e a possibilidade de generalização (CHURCHILL, 1979 e MALHOTRA, 1996). As demais etapas, etapa 6 - coletar mais dados de uma amostra diferente, etapa 7 - avaliar a confiabilidade, a validade e a possibilidade de generalização da escala e etapa 8 - preparar a escala final, do framework apresentado por Churchill (1979), indicam o caminho para que outros pesquisadores possam, com base nos estudos apresentados neste artigo, consolidar uma escala que auxilie as organizações a medir e avaliar o seu relacionamento com os seus diferentes públicos, ou seja, o relacionamento organizacional.

\section{Referências}

ANDERSON, J. C., NARUS, J. A. A model of distributor firm and manufacturer firm working partnerships. Journal of Marketing, 54 (January), 42-58, 1990.

ANDRADE, C. T. S. Para entender relações públicas. 3. ed. São Paulo: Editora Loyola, 2001.

BARDIN, L. Análise de conteúdo. Lisboa/Portugal: Edições 70, 1988.

BERRY, L. Retailers with a future. Marketing Management, 5, 39-46, Spring 1995.

BONFADINI, G. J. 0 relacionamento com públicos como estratégia de comunicação nas organizações. Tese (Doutorado em Comunicação Social) - Pontifícia Universidade Católica do Rio Grande do Sul - PUC-RS, 2007. 175 f.

CHURCHILL, G. A. Jr. A paradigm for developing better measures of marketing constructs. Journal of Marketing Research, 6473, 16 (February, 1979). 
A MENSURAÇ̃̃O DO RELACIONAMENTO ORGANIZACIONAL: CONSTRUÇÃO E VALIDAÇÃO DE UMA ESCALA ORIUNDA DE DUAS ÁREAS DO CONHECIMENTO HUMANO • GERSON JOSÉ BONFADINI • SANDRO LUIS KIRST

COSTA, M. E. B. Grupo Focal. In: DUARTE, J.; BARROS, A. (orgs.) Métodos e técnicas de pesquisa em comunicação. São Paulo: Atlas, 2005

D'AZEVEDO, M. A. Relações públicas: teoria e processo. Porto Alegre: Sulina, 1971.

ENRIQUEZ, E. Vida psíquica e organização In: MOTTA, F. C. P. e FREITAS M. E. Vida psíquica e organização. Rio de Janeiro: Editora FGV, 2000.

FONTENOT, R. J.; WILSON, E. J. Relational exchange: a review of selected models for a prediction matrix of relationship activities. Journal of Business Research, Vol. 39, p. 5-12, 1997.

FORTES, W. G. Relações públicas: processo, funções, tecnologia e estratégias. 2. ed. São Paulo: Summus, 2003.

FRANÇA, F. Públicos: como identificá-los em uma nova visão estratégica. São Caetano do Sul/SP: Difusão, 2004.

GANESAN, S. Determinants of long-term orientation in buyer-seller relationship. Journal of Marketing, Chicago: Vol. 58, N. 2, p. 1, Apr $1994.19 \mathrm{p}$.

GARBARINO, E.; JOHNSON, M. The different roles of satisfaction, trust and commitment in customer relationships. Journal of Marketing, v. 63, n. 2, p. 70-87, 1999.

GRÖNROOS, C. From marketing mix to relationships marketing: towards a paradigm shift in marketing. Management Decision, v. 32, p. $4-20,1994$.

GRUNIG, J. E.; HON, L. Guidelines for measuring relationships in public relations. Gainesville/FI: The Institute for Public Relations, 1999.

GRUNIG, J. E.; HUNT, T. Managing public relations. New York: Harcourt Brace Javonovich College, 1983.

HON, L.; BRUNNER, B. Measuring public relations among students and administrators at the University of Florida. Journal of Communication Management, 6, 3; ABI/Inform Global, Mar 2002. p. 227.

HUNG, C. H. Exploring types of organization-public relationships and their implications for relationship management in public relations. Journal of Public Relations Research, 17(4), 393-425, 2005.

JOSHI, A.W.; STUMP, R.L. The contingent effect of specific asset investments on joint action in manufacturer supplier relationship: an empirical test of the moderating role of reciprocal assets investments, uncertainty and trust. Academy of Marketing Science, p. 1-27, Summer 1999.

KUNSCH, M. M. K. Relações públicas e modernidade: novos paradigmas da comunicação. 2 ed. São Paulo: Summus, 1997.

KUNSCH, M. M. K. Planejamento de relações públicas na comunicação integrada. 9. ed. São Paulo: Summus, 2003.

LEDINGHAM, J. A.; BRUNING, S. D. Relationship management and public relations: dimensions of an organization-public relationship. Public Relations Review, 24, p. 55-65, 1998.

MALHOTRA, N. K. Pesquisa de marketing: uma orientação aplicada. 3. ed. Porto Alegre: Bookman, 2001. Marketing Research: an applied orientation. Prentice Hall, 1996.

MARDSEN, R.; TOWNLEY, B. Introdução: a coruja de minerva: reflexões sobre a teoria e a prática. In: Handbook de Estudos Organizacionais. São Paulo: Atlas, 2001. v. 2, p. 31-60. 
A MENSURAÇ̃̃O DO RELACIONAMENTO ORGANIZACIONAL: CONSTRUÇÃOO E VALIDAÇ̃̃O DE UMA ESCALA ORIUNDA DE DUAS ÁREAS DO CONHECIMENTO HUMANO • GERSON JOSÉ BONFADINI • SANDRO LUIS KIRST

MATTAR, F. N. Pesquisa de marketing. São Paulo: Atlas, 1996. (ed. compacta)

McDANIEL, C.; GATES, R. Pesquisa de marketing. São Paulo: Pioneira Thomson Learning, 2003.

MOORMAN, C.; ZALTMAN, G. \& DESHPANDÉ, R. Relationships between providers and users of market research: the dynamics of trust within and between organizations. Journal of Marketing Research, v. 29, n. 3, p. 314-28, ago. 1992.

MORGAN, R. M.; HUNT, S. D. The commitment - trust theory of relationship marketing. Journal of Marketing, Jul. 1994.

NOOTEBOOM, B.; BERGER, H. \& NOORDERHAVEN, N. G. Effects of trust and governance on relational risk. Academy of Management Journal, 40 (2), 308-338, 1997.

OLIVER, Richard L. Satisfaction: a behavioral perspective on the consumer. New York: Irwin/McGraw-Hill, 1997.

REA, L. M.; PARKER, R. A. Metodologia da pesquisa: do planejamento à execução. São Paulo: Pioneira Thomson Learning, 2002.

SCHEIN, E. H. Organizational psychology. Englewood Cliffs, N. J.: Prentice-Hall, 1980.

SCHWARTZ, S. H. Are there universal aspects in the structure and content of human values? Journal of Social Issues, v. 50, p.19-45, 1994.

SIMÕES, R. J. P. Relações públicas: função política. 5. ed. São Paulo: Summus, 1995.

Relações públicas e micropolítica. São Paulo: Summus, 2001.

SIRDESHMUKH, D.; SINGH, J. \& SABOL, B. Consumer trust, value, and loyalty in relational exchanges. Journal of Marketing, v. 66, p. 15-37, January, 2002.

WHERRY, R. J. Contributions to correlation analysis. Orlando: Academic Press, 1984.

WILSON. E.; VLOSKY, R. Partnering relationships activities: building theory from case study research. Journal of Business Research, May, 1997.

YANG, S. U.; GRUNIG J. E. Decomposing organizational reputation: the effects of organization-public relationship outcomes on cognitive representations of organizations and evaluations of organizational performance. Journal of Communication Management, 9, 4; ABI/Inform Global, p. 305, 2005.

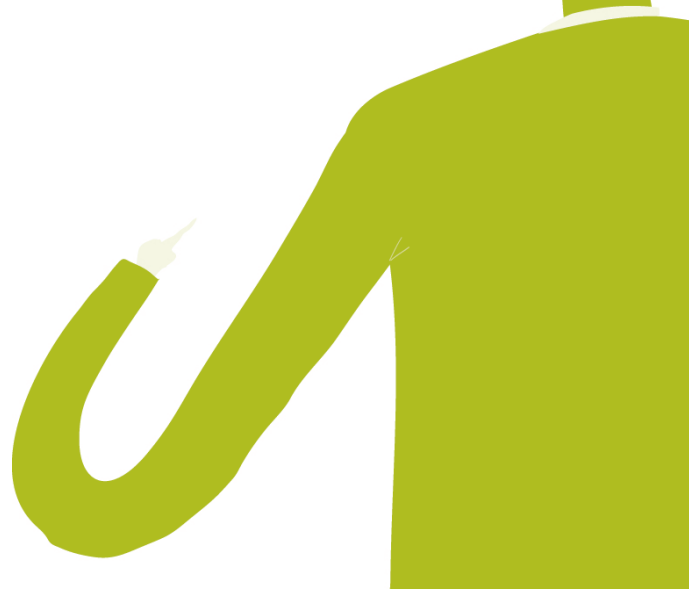

